\title{
Multiple Isolated Intracranial Tuberculomas Masquerading as Brain Metastases on Radiological Imaging: Success of a Therapeutic Trial
} Junaid Nabi*

Department of Surgery, Shaheed Suhrawardy Medical College and Hospital, Bangladesh

\begin{abstract}
Tuberculosis is endemic in Bangladesh. It remains a resilient public health concern. Reports on presentation and management of intracranial tuberculoma from Bangladesh are sparse. Despite being potentially remediable, tuberculomas are still a cause of significant morbidity and mortality in developing nations. Intracranial tuberculomas can occur as solitary or multiple lesions. Multiple isolated intracranial tuberculomas are rare, and difficult to discern from brain metastases. A unique case of intracranial tuberculomas is presented, which masqueraded as brain metastases on imaging studies and was diagnosed and treated with a trial of antitubercular chemotherapy. A 43-year-old Bengali male presented to the OPD with headaches and blurring of vision for four months. He was provided symptomatic treatment at his regional hospital, which did not abate his condition and was later referred to our center when he developed severe vomiting. Routine investigations including chest radiograph were clean. CT scan showed bilateral edema in cerebral hemispheres and MRI revealed rounded signal change areas of right frontal lobe and left parietal lobe posteriorly, strongly suggestive of brain metastases. Patient declined biopsy. In view of his past history, a full course of antitubercular chemotherapy was prescribed. Following the medication, patient's condition improved and at 24 months follow-up, there was complete resolution of the lesion.
\end{abstract}

Keywords: Intracranial tuberculoma; Brain metastases; Magnetic resonance imaging; Antitubercular chemotherapy

\section{Introduction}

Multiple isolated intracranial tuberculomas are rare. Intracranial tuberculomas (ICTs) are manifestation of infection caused by Mycobacterium tuberculosis, and usually result from hematogenous spread of infection from a primary focus elsewhere in the body, which may be clinically evident or dormant. Tuberculosis, being endemic in the Indian subcontinent, presents in multifarious forms. Intracranial tuberculomas accounts for $10 \%$ to $30 \%$ of all intracranial masses in the developing world [1]. Although being potentially curable and many modern methods being available for early detection, intracranial tuberculomas are still associated with high morbidity and mortality [2]. Clinical manifestations are atypical and non-specific and it has been reported that evidence of systemic tuberculosis or exposure to the disease may be absent in $70 \%$ of the cases [3], which may lead to delayed diagnosis. In developing countries, a high degree of suspicion is warranted to prevent late diagnosis. Fortunately, imaging modalities such as computed tomography (CT) have immensely improved the accuracy of diagnosing intracranial tuberculoma and has a role in elucidating information about the response to chemotherapy $[4,5]$, when required. However, neoplastic, fungal, and parasitic diseases may show similar features on CT scan [6]. Role of CT-guided brain biopsy is unclear [7]. Magnetic resonance imaging (MRI), on the other hand, is superior to CT scan in demonstrating the extent of the lesion as features of tuberculomas are more distinct from those of abscesses, metastates, and gliomas [8]. Although, tuberculosis is endemic in Bangladesh, there is a scarcity of reports on intracranial tuberculomas. This report presents an interesting case of multiple intracranial tuberculomas which presented with prolonged nonspecific symptoms and mimicked brain metastases on radiological imaging and discusses the imaging features of intracranial tuberculoma along with the role of anti-tubercular chemotherapy as a modality for diagnosis in a limited resource setting.

\section{Case Presentation}

A 43-year-old Bengali male was admitted to our hospital with chief complaints of headache and blurring of vision. The patient had been well till four months before admission, when he developed anorexia and malaise. After one month, he developed headache and two weeks later was seen at a local hospital where he was prescribed analgesics after no abnormality was detected on physical examination, chest radiography, and routine laboratory examinations. He was referred to our hospital after he developed vomiting and blurring of vision as the regional hospital did not have CT scan or MRI facilities. On admission, the patient also complained of weight loss and night sweating. The headache was moderate in severity, dull aching in character, intermittent in nature, usually localized in the front of his head but often radiated to the sides and back. He had noticed that the pain was more on waking up in the morning, he also reported that after taking analgesics the pain would subside but his head would feel "heavy". Family history revealed that his father was a patient of tuberculosis and had not completed the prescribed course of anti-tubercular therapy.

On physical examination, he was generally wasted. He had an oral temperature of $38.2^{\circ} \mathrm{C}$, pulse rate of 84 per minute, respiratory rate of 17 per minute and blood pressure of $125 / 82 \mathrm{mmHg}$. He was found to have papilloedema but otherwise no abnormality was detected. Blood tests revealed elevated ESR ( $45 \mathrm{~mm}$ in first hour, Westergren Method) and intradermal tuberculin test (Mantoux test) reported an induration of $16 \mathrm{~mm}$. No abnormalities were detected on his chest and skull $\mathrm{x}$-rays.

Brain computed tomography (CT) scan reported cerebral edema in

*Corresponding author: Junaid Nabi, MBBS, Department of Surgery, Shaheed Suhrawardy Medical College and Hospital, Sher-e-Bangla Nagar, Dhaka-1207, Dhaka, Bangladesh, E-mail: junaidnabi07@gmail.com

Received September 19, 2015; Accepted October 23, 2015; Published October 30, 2015

Citation: Nabi J (2015) Multiple Isolated Intracranial Tuberculomas Masquerading as Brain Metastases on Radiological Imaging: Success of a Therapeutic Trial. Arch Surg Oncol 1: 102. doi: 10.4172/2471-2671.1000102

Copyright: (c) 2015 Nabi J. This is an open-access article distributed under the terms of the Creative Commons Attribution License, which permits unrestricted use, distribution, and reproduction in any medium, provided the original author and source are credited. 
Citation: Nabi J (2015) Multiple Isolated Intracranial Tuberculomas Masquerading as Brain Metastases on Radiological Imaging: Success of a Therapeutic Trial. Arch Surg Oncol 1: 102. doi: 10.4172/2471-2671.1000102

both hemispheres. Hypodense areas were noted in left parietal and right frontal lobes in the supratentorial region with poorly defined margins. No hydrocephalus was noted. Magnetic resonance imaging (MRI) of the brain revealed rounded signal change areas of right frontal lobe and left parietal lobe posteriorly (T1W1-hypointense, T2W1-hyperintense, FLAIR-hypointense and after gadolinium contrast I/V-strong contrast ring enhancement) (Figure 1). There was also perilesional edema around the lesions with signal change (T1W1-hypointense, T2W1 and FLAIR-hyperintense and after gadolinium contrast I/V-no contrast enhancement). There was effacement of the anterior horn of the right lateral ventricle and midline shift to left side anteriorly. There was also effacement of the posterior horn of the left lateral ventricle with minimum shift to the right posteriorly (Figure 2). Following CT and MRI scan, a diagnosis of intracranial space occupying lesion suggestive of brain tuberculoma was made. The patient was advised to undergo a CT-guided stereotactic biopsy to rule out brain metastases, which he declined. Based on the radiological features of the intracranial lesions, exposure of the patient to tuberculosis and the endemic nature of the disease in Bangladesh, diagnosis of intracranial tuberculoma were made.

The patient was immediately started on long term anti-tubercular chemotherapy. The regimen consisted of Isoniazid, Rifampicin, Ethambutol, and Pyrazinamide with appropriate dosages according to the weight of the patient. Vitamin-B6 (Pyridoxine) and Dexamethasone were also added to the regimen. After three weeks the patient became afebrile. The patient was discharged from the hospital with good general condition and moderate decrease in headaches. Ethambutol and Pyrazinamide were discontinued after two months and treatment was continued by Isoniazid and Rifampicin. Follow up CT examination at 8 months revealed intracranial tuberculomas were still present but smaller in size and without enhancement. At 24 months, he remained asymptomatic and CT showed complete resolution of the lesion (Figure 3).
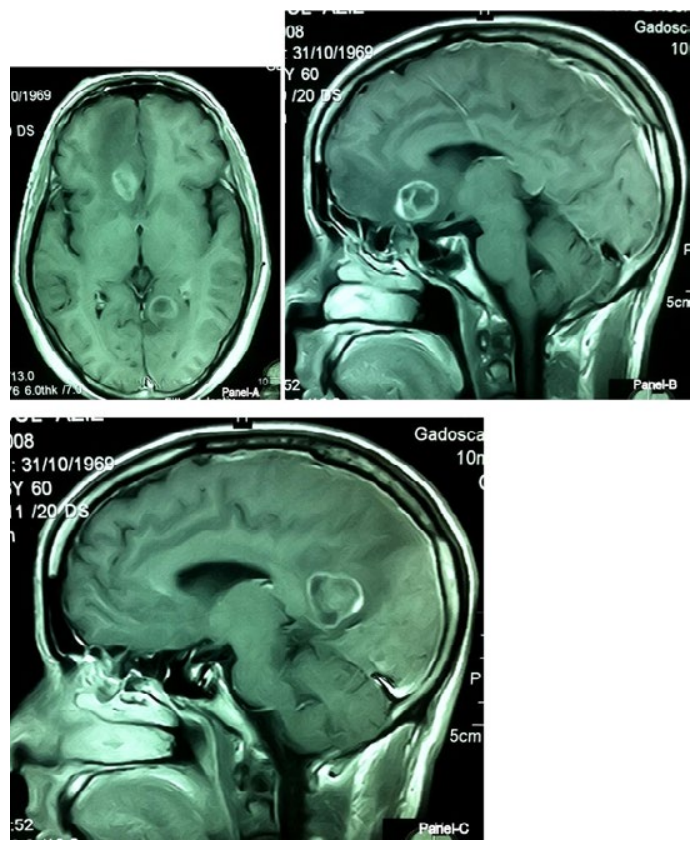

Figure 1: MRI appearance of intracranial tuberculomas showing signal change areas of right frontal lobe and left parietal lobe posteriorly (T1W1hypointense, T2W1-hyperintense, FLAIR-hypointense and after gadolinium contrast I/V-strong contrast ring enhancement).
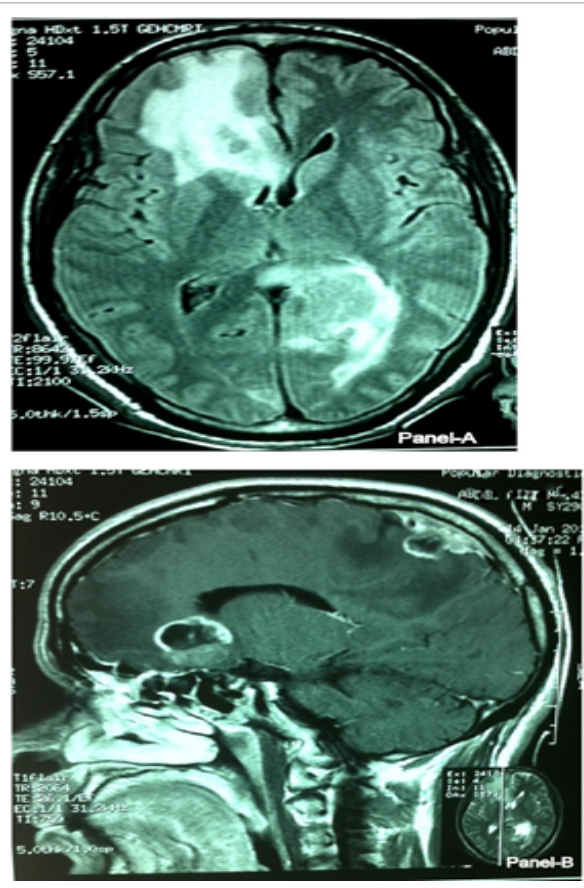

Figure 2: Effacement of the anterior horn of the right lateral ventricle and midline shift to left side anteriorly can be observed along with effacement of the posterior horn of the left lateral ventricle with minimum shift to the right posteriorly.

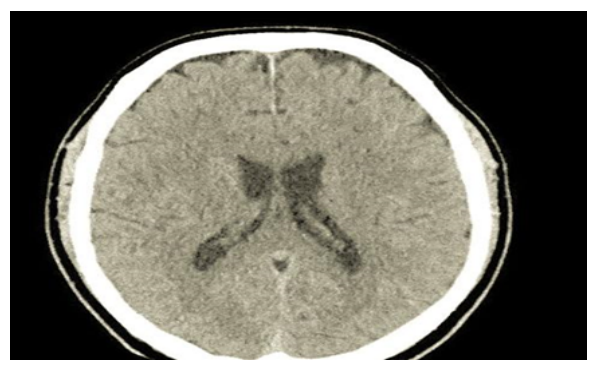

Figure 3: Plain Computed Tomography (CT) Scan completed at 24 months post-chemotherapy shows the normal appearance of the second and fourth ventricles.

\section{Discussion}

Intracranial tuberculomas form a significant proportion of intracranial space-occupying lesions (ICSOLs) presented to physicians in the developing countries [1] and lead to significant morbidity and mortality [2]. Early recognition of the lesion on imaging and prompt treatment may be critical to management of these patients. In fact, the most important physician controlled factor which can influence the prospect of recovery and result in avoidance of neurological deficits as a sequel, has been reported to be the promptness with which the treatment was initiated $[9,10]$. Tuberculomas mimicking gliomas is not unheard of: Brismar has compiled a lucid report on many cases, which were sent for tumor resection but ended up being diagnosed as tuberculomas [11]. Malignant gliomas remain a stubborn disease with unpropitious prognosis [12], and CT-guided stereotactic brain biopsy does not always yield the correct diagnosis, as the needle is not able to penetrate the dense capsule of the tuberculoma [7]. In such cases, open biopsy of the lesion is recommended [7]; however, it is not feasible option in a limited resource setting such as ours. 
Citation: Nabi J (2015) Multiple Isolated Intracranial Tuberculomas Masquerading as Brain Metastases on Radiological Imaging: Success of a Therapeutic Trial. Arch Surg Oncol 1: 102. doi: 10.4172/2471-2671.1000102

Page 3 of 3

Tuberculomas in the CNS may present as solitary or multiple lesions; solitary lesions are seen more often in clinical practice. Brain tuberculomas usually occur in isolation, with only $30 \%$ of patients having a positive chest radiograph [13]. In an endemic area such as Bangladesh, it's imperative for clinicians to suspect tuberculosis even when the chest radiographs are clean but the symptoms are suggestive, such as in our case. With the advances in CT scan and MRI, the incidence and the ability to diagnose brain tuberculomas at an early stage has increased, however, such modalities are not readily available in all developing nations. Some studies contend that imaging findings of brain tuberculomas may be non-specific $[7,11,12]$ and differentiating them from other ICSOLs such as metastases, cysticercosis, abscesses and gliomas is important. There does exist a relationship between the appearance of brain tuberculomas on the MR imaging and their histology [9], although it varies on the stage of the lesion. Brain tuberculomas which consist of non-caseating granulomas are hypo-intense compared to normal brain tissue on T1-weighted images (T1WI) and hyper-intense on T2-weighted images (T2WI). The central solid core of caseating granulomas shows iso-intense and hypo-intense signals on T1WI and T2WI, respectively. The middle layer, which consists of Langhans giant cells and epithelioid cells, is edematous. This layer shows hypo-intense and hyper-intense signals on T1WI and T2WI, respectively. The outer collagenous capsule shows iso-intense and hypo-intense signals on T1WI and T2WI, respectively. The middle layer is the only one showing ring-enhancing lesion with gadolinium injection on T1WI [14]. Thus producing a "target sign" which is described as pathognomonic for tuberculomas [15]. MR spectroscopy has also been found very useful in differentiating between tuberculomas and other ICSOLs; MR spectroscopy is characterized by slight decrease in NAA/Cho and significant decrease in NAA/Cr [14]. Surgical excision or stereotactic biopsy, although available at only few centers in the country, would have been an ideal option to establish diagnosis but due to economic constraints the patient declined. However, since tuberculous bacilli are not always present in CSF or even the excised tissue [16], negative results do not rule out the presence of tuberculous infection. Therapeutic trial with multidrug chemotherapy was the only option left to establish diagnosis and alleviate the patient's symptoms. There is, however, a caveat in the therapeutic chemotherapy which is a sporadic occurrence, but clinicians should be aware of this phenomenon: "paradoxical expansion". This phenomenon occurs when in spite of recommended dosage of chemotherapy, there is abnormal expansion of the tuberculomas or appearance of new small ones [17] which may conflate the diagnosis and result in unnecessary surgery. Less than 100 cases [18] have been reported in the literature and even though it warrants caution from the attending physician, continuation of the chemotherapy for longer duration along with dexamethasone is recommended which usually results in abated lesions. Our patient was placed on appropriate dosage of Dexamethasone from the start of the regimen which most probably explains the absence of this rare phenomenon. Dexamethasone is, as such, highly recommended in such scenarios. Tuberculomas of the brain should be treated with longer-than-usual dosage regimens [17] to prevent the "paradoxical expansion".

\section{Conclusion}

In an endemic setting such as Bangladesh, tuberculoma should always be considered in the differential diagnosis of ICSOL. Diagnosis of brain tuberculoma is difficult due to variations in imaging presentations; symptoms and initial work up may be non-specific and economic constraints may be a hurdle to establish diagnosis. Brain tuberculoma often mimics the appearance and behavior of brain metastases and the only definite way to distinguish between these two lesions is by excision biopsy, or by treating the patient with a complete course of anti-tubercular chemotherapy.

\section{Consent}

A written informed consent was obtained from the patient for publication of this case report.

\section{References}

1. Dastur HM, Desai AD (1965) A comparative study of brain tuberculomas and gliomas based upon 107 case records of each. Brain 88: 375-396.

2. Arvanitakis Z, Long RL, Hershfield ES, Manfreda J, Kabani A, et al. (1998) $M$. tuberculosis molecular variation in CNS infection: evidence for straindependent neurovirulence. Neurology 50: 1827-1832.

3. DeAngelis LM (1981) Intracranial tuberculoma: case report and review of the literature. Neurology 31: 1133-1136.

4. Whelan MA, Stern J (1981) Intracranial tuberculoma. Radiology 138: 75-81.

5. Vengsarkar US, Pisipaty RP, Parekh B, Panchal VG, Shetty MN (1986) Intracranial tuberculoma and the CT scan. J Neurosurg 64: 568-574.

6. Naim-Ur-Rahman (1987) Intracranial tuberculomas: Diagnosis and management. Acta Neurochir (Wien) 88: 109-115.

7. Haddadian K, Rezaei O, Samadian M (2005) Multiple brain tuberculomas and role of open brain biopsy: a case report and review. The Internet Journal of Infectious Diseases 4.

8. Gupta RK, Jena A, Singh AK, Sharma A, Puri V, et al. (1990) Role of magnetic resonance $(M R)$ in the diagnosis and management of intracranial tuberculomas. Clin Radiol 41: 120-127.

9. Kim TK, Chang KH, Kim CJ, Goo JM, Kook MC et al. (1995) Intracranial tuberculoma: Comparison of MR with pathologic findings. AJNR Am J Neuroradiol 16: 1903-1908.

10. Sheller JR, Des Prez RM (1986) CNS tuberculosis. Neurol Clin 4: 143-158.

11. Brismar J, Hugosson C, Larsson SG, Lundstedt C, Nyman R (1996) Imaging of tuberculosis. III. Tuberculosis as a mimicker of brain tumour. Acta Radiol 37: 496-505.

12. Omuro A, DeAngelis LM (2013) Glioblastoma and other malignant gliomas: A clinical review. JAMA 310: 1842-1850.

13. Jinkins JR (1991) Computed tomography of intracranial tuberculosis Neuroradiology 33: 126-135.

14. Gupta RK, Vatsal DK, Husain N, Chawla S, Prasad KN, et al. (2001) Differentiation of tuberculous from pyogenic brain abscesses with in vivo proton MR spectroscopy and magnetization transfer MR imaging. AJNR Am J Neuroradiol 22: 1503-1509.

15. Whiteman ML (1997) Neuroimaging of central nervous system tuberculosis in HIV-infected patients. Neuroimaging Clin N Am 7: 199-214.

16. Yanardag H, Uygun S, Yumuk V, Caner M, Canbaz B (2005) Cerebral tuberculosis mimicking intracranial tumour. Singapore Med J 46: 731-733.

17. Reiser M, Fätkenheuer G, Diehl V (1997) Paradoxical expansion of intracranial tuberculomas during chemotherapy. J Infect 35: 88-90.

18. Hejazi N, Hassler W (1997) Multiple intracranial tuberculomas with atypical response to tuberculostatic chemotherapy: Literature review and a case report. Acta Neurochir (Wien) 139: 194-202.

Citation: Nabi J (2015) Multiple Isolated Intracranial Tuberculomas Masquerading as Brain Metastases on Radiological Imaging: Success of a Therapeutic Trial. Arch Surg Oncol 1: 102. doi: 10.4172/2471-2671.1000102 